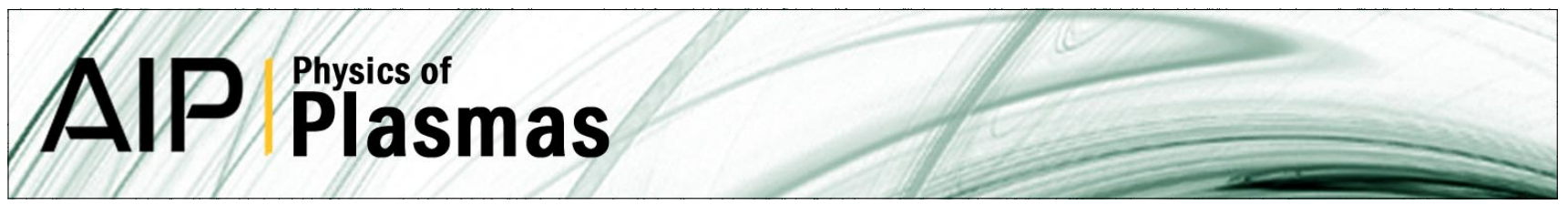

\title{
On the breaking of a plasma wave in a thermal plasma. II. Electromagnetic wave interaction with the breaking plasma wave
}

Sergei V. Bulanov, Timur Zh. Esirkepov, Masaki Kando, James K. Koga, Alexander S. Pirozhkov et al.

Citation: Phys. Plasmas 19, 113103 (2012); doi: 10.1063/1.4764056

View online: http://dx.doi.org/10.1063/1.4764056

View Table of Contents: http://pop.aip.org/resource/1/PHPAEN/v19/i11

Published by the American Institute of Physics.

\section{Related Articles}

Electro-diffusion in a plasma with two ion species

Phys. Plasmas 19, 082709 (2012)

Thermodynamic and transport properties of two-temperature SF6 plasmas

Phys. Plasmas 19, 083506 (2012)

A database for equations of state and resistivities measurements in the warm dense matter regime Phys. Plasmas 19, 082702 (2012)

Thermodynamic bounds on nonlinear electrostatic perturbations in intense charged particle beams Phys. Plasmas 19, 073113 (2012)

Electronic excitation and isentropic coefficients of high temperature planetary atmosphere plasmas Phys. Plasmas 19, 072115 (2012)

\section{Additional information on Phys. Plasmas}

Journal Homepage: http://pop.aip.org/

Journal Information: http://pop.aip.org/about/about_the_journal

Top downloads: http://pop.aip.org/features/most_downloaded

Information for Authors: http://pop.aip.org/authors

\section{ADVERTISEMENT}

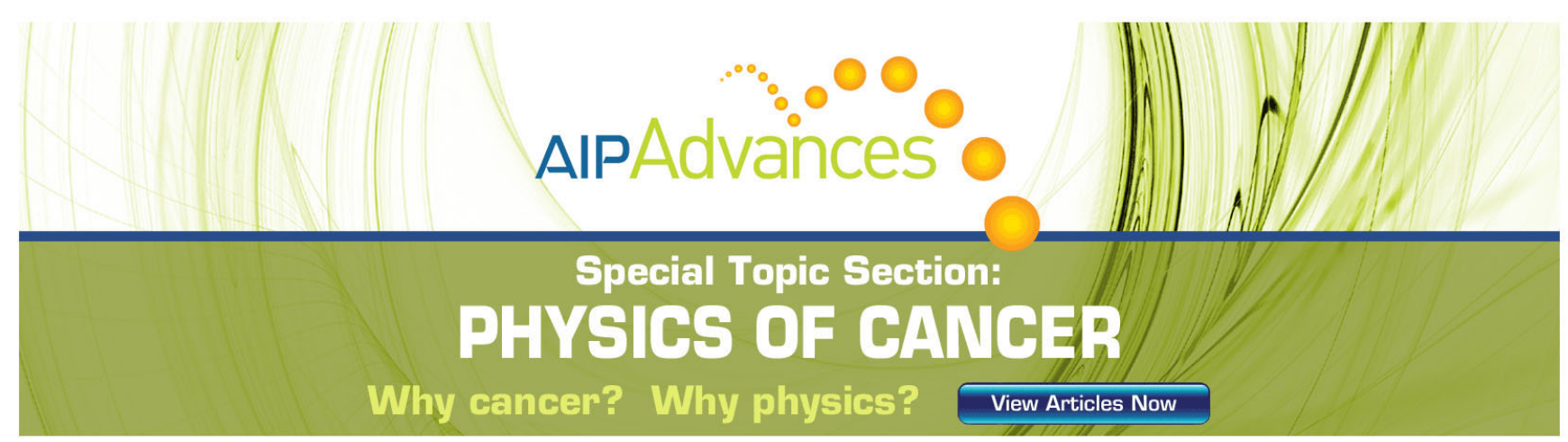




\title{
On the breaking of a plasma wave in a thermal plasma. II. Electromagnetic wave interaction with the breaking plasma wave
}

\author{
Sergei V. Bulanov, ${ }^{1, a)}$ Timur Zh. Esirkepov, ${ }^{1}$ Masaki Kando, ${ }^{1}$ James K. Koga, ${ }^{1}$ \\ Alexander S. Pirozhkov, ${ }^{1}$ Tatsufumi Nakamura, ${ }^{1}$ Stepan S. Bulanov, $\left.{ }^{2, b}\right)$ \\ Carl B. Schroeder, ${ }^{3}$ Eric Esarey, ${ }^{3}$ Francesco Califano, ${ }^{4}$ and Francesco Pegoraro ${ }^{4}$ \\ ${ }^{1}$ QuBS, Japan Atomic Energy Agency, 1-8-7 Umemidai, Kizugawa, Kyoto 619-0215, Japan \\ ${ }^{2}$ University of California, Berkeley, California 94720, USA \\ ${ }^{3}$ Lawrence Berkeley National Laboratory, Berkeley, California 94720, USA \\ ${ }^{4}$ Physics Department, University of Pisa, Pisa 56127, Italy
}

(Received 8 February 2012; accepted 10 October 2012; published online 8 November 2012)

In thermal plasma, the structure of the density singularity formed in a relativistically large amplitude plasma wave close to the wavebreaking limit leads to a refraction coefficient with discontinuous spatial derivatives. This results in a non-exponentially small above-barrier reflection of an electromagnetic wave interacting with the nonlinear plasma wave. (C) 2012 American Institute of Physics. [http://dx.doi.org/10.1063/1.4764056]

\section{INTRODUCTION}

In the first part of our paper, ${ }^{1}$ extending an approach formulated in Ref. 2 to the relativistic limit, we have studied systematically the structure of the singularities formed in a relativistically large amplitude plasma wave close to the wavebreaking in a thermal plasma. We have shown that the electron density distribution in the breaking wave typically has a "peakon" form with a discontinuous spatial derivative, similar to the profiles of nonlinear water waves ${ }^{3-5}$ and that in the above breaking limit the derivative becomes infinite. This results in a finite reflectivity of an electromagnetic (EM) wave interacting with nonlinear plasma waves. This is an important property, in particular, because nonlinear Langmuir waves play a key role in the "relativistic flying mirror" concept. ${ }^{6-11}$ In this concept, very high density electron shells are formed in the nonlinear wake wave generated by an ultrashort laser pulse propagating in an underdense plasma with a speed close to the speed of light in vacuum. The shells act as mirrors flying with relativistic velocity. When they reflect a counterpropagating electromagnetic pulse, the pulse is compressed, its frequency is upshifted, and its intensity is increased. It is the singularity in the electron density distribution that affords a high efficiency in the reflection of a portion of the counterpropagating electromagnetic pulse. If the Langmuir wave is far below the wave-breaking threshold, its reflectivity is exponentially small. For a nonlinear Langmuir wave, the singularity formed in the electron density breaks the geometric optics approximation and leads to a reflection coefficient that is not exponentially small. ${ }^{6,12}$

In the present paper, we address the problem of the interaction of an electromagnetic wave with a nonlinear plasma wave which is of interest for the "photon accelerator" concept ${ }^{13,14}$ and for the "relativistic flying mirror" paradigm. ${ }^{6-11} \mathrm{We}$ calculate the reflection coefficients of an electromagnetic wave at the

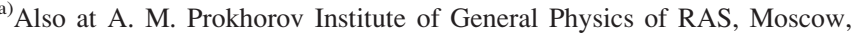
Russia.

b) Also at Institute of Theoretical and Experimental Physics, Moscow 117218, Russia.
}

singularities of the electron density in the most typical regimes of a strongly nonlinear wave breaking in thermal plasmas.

\section{ELECTROMAGNETIC WAVE REFLECTION BY THE ELECTRON DENSITY MODULATED IN THE BREAKING WAVE}

As we have seen in the first part of our paper, ${ }^{1}$ in a strongly nonlinear wake wave the electron density is modulated and forms thin shells (singularities or caustics in the plasma flow) moving with velocity $\beta_{\mathrm{ph}}$. In the Introduction, in a way of Refs. 6-11, we have discussed how a counterpropagating electromagnetic wave can be partially reflected from these density shells which play the role of relativistic mirrors. While in the case of a cold plasma, the electron density at the singularity tends to infinity (see Eq. (59) of Part $\mathrm{I}^{1}$ and Refs. 7 and 12), in a thermal plasma the density is limited by the expressions given by Eqs. (41) and (42) of Part I. ${ }^{1}$ Although in this case the density profile is described by a continuous function, its derivative is discontinuous. This discontinuity results in the breaking of the geometric optics approximation and leads to a reflectivity that is not exponentially small.

In order to calculate the reflection coefficient, we consider the interaction of an electromagnetic wave with the electron density shell formed at the breaking point of a Langmuir wave in a thermal plasma similarly to what has been done in Refs. 6 and 12. The electromagnetic wave, described by the $z$ component of the vector potential $A_{z}(x, y, t)$, evolves according to the linearized wave equation

$$
\partial_{t t} A_{z}-c^{2}\left(\partial_{x x} A_{z}+\partial_{y y} A_{z}\right)+\Omega_{p e}^{2}\left(x-v_{\mathrm{ph}} t\right) A_{z}=0,
$$

where the square of the local Langmuir frequency is

$$
\Omega_{p e}^{2}(X)=\frac{4 \pi e^{2}}{m_{e}} \int_{-\infty}^{+\infty} \frac{f_{e}(p, X) \mathrm{d} p}{\sqrt{1+\left(p / m_{e} c\right)^{2}}} .
$$

The last term in the 1.h.s. of Eq. (1) is the $z$-component of the electric current density generated by the electromagnetic 
wave in a plasma with the electron distribution function $f_{e}(p, X)$. In the limit $\gamma_{\mathrm{ph}} \Delta p_{0} \ll 1$ for the electromagnetic wave frequency larger than the Langmuir frequency calculated for the maximal electron density, $\omega \gg \omega_{\mathrm{pe}}\left(2 \gamma_{\mathrm{ph}}\right.$ $\left(\Delta p_{0}\right)^{1 / 4}$, we can neglect the finite temperature effects on the electromagnetic wave dispersion, which have been analyzed in Ref. 15, in the limit of homogeneous, stationary plasmas. For the water-bag distribution function

$$
f_{e}(p, X)=n_{0} \theta\left(p-p_{-}(X)\right) \theta\left(p_{+}(X)-p\right) / \Delta p_{0},
$$

$\Omega_{p e}^{2}(X)$ takes the form

$$
\Omega_{p e}^{2}(X)=\omega_{p e}^{2} \frac{1}{\Delta p_{0}} \ln \left(\frac{p_{+}(X)+\sqrt{1+p_{+}(X)^{2}}}{p_{-}(X)+\sqrt{1+p_{-}(X)^{2}}}\right),
$$

where $\omega_{p e}^{2}=4 \pi n_{0} e^{2} / m_{e}, p_{ \pm}(X)$ and $\Delta p_{0}$ are normalized on $m_{e} c$.

The wake wave modulates the electron density and temperature increasing them in the compression regions and decreasing them in the rarefaction regions. In Fig. 1, we illustrate the dependence of $\Omega_{p e}(X) / \omega_{p e}$ on $X$ for the parameters of a wakewave corresponding to $\Delta p_{0}=0.1$ and $E_{\max }=2.3$ at $X=15$ and for $\beta_{\mathrm{ph}}=0.992$.

From Eqs. (35) and (59) of Part $\mathrm{I}^{1}$ in the ultrarelativistic case, $\beta_{\mathrm{ph}} \approx 1$, using Eq. (4) we find for a relatively cold distribution such that $p_{-} \ll 1$ that near the wavebreaking point $\Omega_{p e}^{2}(X)$ is given by

$$
\Omega_{p e}^{2}(X) \approx \frac{\omega_{p e}^{2}}{\gamma_{\mathrm{ph}}}-\frac{\omega_{p e}^{2} \sqrt{n_{\mathrm{br}} \gamma_{\mathrm{ph}}}}{\Delta p_{0}}|X| .
$$

The propagation of a sufficiently short electromagnetic wave packet in the plasma with electron density modulated by the Langmuir wave can be described within the framework of the geometric optics approximation. The electromagnetic wave is represented as a particle ("photon") with coordinate $x$ and momentum $\mathrm{k}$ (wave vector). The interaction of a "photon" with a Langmuir wave that propagates with a relativistic phase velocity $v_{\mathrm{ph}} \approx c$ can be accompanied by a substantial frequency upshift called "photon acceleration."13,14,16,17 Using the dispersion equation

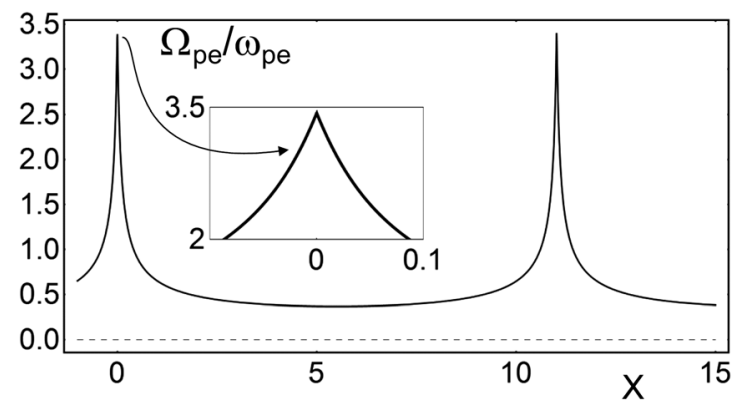

FIG. 1. Dependence of the frequency ratio $\Omega_{p e} / \omega_{p e}$ on the coordinate $X$ for the parameters of a wakewave corresponding to $\Delta p_{0}=0.1$ and $E_{\max }=2.3$ at $X=15$ and for $\beta_{\mathrm{ph}}=0.992$. In the inset, the ratio $\Omega_{p e}(X) / \omega_{p e}$ is shown in the vicinity of the maximum.

$$
\omega(x, \mathbf{k} ; t)=\sqrt{k^{2} c^{2}+\Omega_{p e}^{2}\left(x-v_{\mathrm{ph}} t\right)},
$$

where $k^{2}=k_{\|}^{2}+k_{\perp}^{2}$ with $k_{\|}$and $k_{\perp}$ the wave vector components parallel and perpendicular to the propagation direction of the Langmuir wave, we obtain the "photon" Hamiltonian function which depends on the canonical variables $X=$ $x-v_{\mathrm{ph}} t$ and $k_{\|}$(see Ref. 18) as

$$
\mathcal{H}_{\text {photon }}\left(X, k_{\|}\right)=\sqrt{k_{\|}^{2} c^{2}+\Omega_{p e}^{2}(X)}-\beta_{\mathrm{ph}} k_{\|} c .
$$

The transverse component of the wave vector is constant $k_{\perp}=k_{\perp, 0}$ and $k_{\perp, 0}=0$ is assumed for the sake of simplicity. The phase portrait of the photon for $\Omega_{p e}(X)$ given by Eq. (4) for the parameters corresponding to Fig. 1 is shown in Fig. 2.

Along an orbit corresponding to the value $\mathcal{H}_{\text {photon }}\left(X, k_{\|}\right)$ $=\mathcal{H}_{\text {photon }}\left(X_{0}, k_{\|, 0}\right)=\mathcal{H}_{\text {photon }, 0}$ of the Hamiltonian (7), the photon frequency is given by

$$
\omega=\gamma_{\mathrm{ph}}^{2} \mathcal{H}_{\text {photon }, 0}\left[1 \pm \beta_{\mathrm{ph}} \sqrt{1-\frac{\Omega_{p e}^{2}(X)}{\mathcal{H}_{\text {photon }, 0} \gamma_{\mathrm{ph}}^{2}}}\right] .
$$

Photons, for which $\mathcal{H}_{\text {photon, } 0}<\max \left\{\Omega_{p e} / \gamma_{\mathrm{ph}}\right\}$ are trapped inside the region enclosed by the separatrix. Along the orbit, their frequency changes between $\omega_{\max }$ and $\omega_{\min }$ corresponding to the plus and minus signs in the r.h.s. of Eq. (8) at the minimum of $\Omega_{p e}(X)$. We note here that under conditions typical for laser-plasma interaction, the time required for photon bouncing between subsequent electron density maxima could be larger than the laser pulse energy depletion time, as discussed at the end of Sec. III. Photons with $\mathcal{H}_{\text {photon }, 0}>\max \left\{\Omega_{p e} / \gamma_{\text {ph }}\right\}$ are not trapped and for them the sign in the r.h.s. of Eq. (8) is "+." For trajectories far above the separatrix, the photon frequency variations are relatively weak.

A sufficiently strong wakefield can reflect a counterpropagating photon, $k_{\|, 0}<0$ due to above-barrier reflection

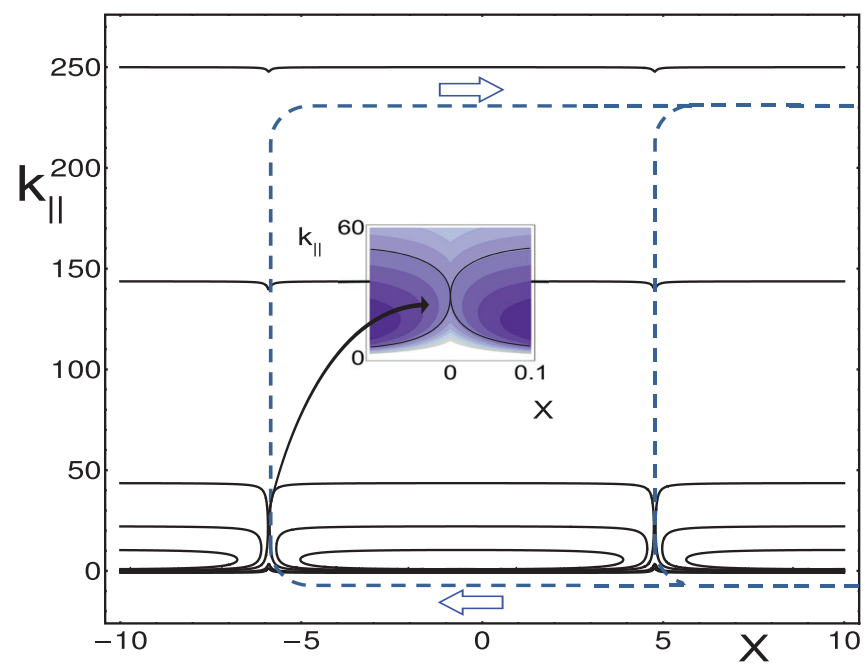

FIG. 2. Photon phase portrait for the parameters of a nonlinear Langmuir wave corresponding to Fig. 1. The dashed line corresponds to the trajectory of photons that have appeared due to the over-barrier reflection at the crest of the breaking wave. In the inset, the photon trajectories in the vicinity of the saddle point are shown. 
(this trajectory is shown in Fig. 2 by a dashed line). Such a photon acquires a frequency

$$
\omega=\omega_{0} \frac{1+\beta_{\mathrm{ph}}}{1-\beta_{\mathrm{ph}}}
$$

according to the Einstein formula for the frequency of the electromagnetic wave reflected by a relativistic mirror. ${ }^{19}$ The geometric optics approximation fails when the wakefield is close to wave breaking and this provides the appropriate conditions for a not exponentially weak wave scattering.

\section{SCATTERING OF AN ELECTROMAGNETIC WAVE AT THE BREAKING WAKE WAVE}

\section{A. Reflectivity of nonlinear wake wave}

In order to find the reflectivity of the nonlinear wake wave, we perform a Lorentz transformation to the frame of reference moving with the phase velocity of the Langmuir wave. In the boosted frame, for the electromagnetic wave interacting with the nonlinear Langmuir wave Eq. (1) can be written as

$$
\frac{d^{2} a(\zeta)}{d \zeta^{2}}+q^{2}(\zeta) a(\zeta)=0
$$

with

$$
a(\zeta)=\frac{e A_{z}}{m_{e} c^{2}} \exp \left[-i\left(\omega^{\prime} t^{\prime}-k_{y} y\right)\right]
$$

In the neighbourhood of the breaking point, $q^{2}(\zeta)$ can be written as

$$
q^{2}(\zeta)=s^{2}+\nu(\zeta)
$$

Here,

$$
s^{2}=\frac{\omega^{\prime 2}}{c^{2}}-k_{y}^{2} \quad \text { and } \quad \nu(\zeta)=\omega_{p e}^{2}(\zeta) / c^{2}
$$

$\zeta=X \gamma_{\mathrm{ph}}, t^{\prime}, k^{\prime}, \omega^{\prime}$ are the coordinate, time, the wave number, and frequency in the boosted frame of reference.

We seek for the solution to the above-barrier scattering problem represented by Eq. (10) writing its solution in the form (see Refs. 12, 20, and 21)

$$
a(\zeta)=\frac{1}{\sqrt{q(\zeta)}}\left[b_{+} \exp (i W(\zeta))+b_{-} \exp (-i W(\zeta))\right]
$$

where the phase integral is defined as

$$
W(\zeta)=\int_{0}^{\zeta} q\left(\zeta^{\prime}\right) d \zeta^{\prime}
$$

The above-barrier scattering geometry is illustrated in Fig. 3. For constant $b_{+}$and $b_{-}$, Eq. (14) corresponds to the "WKB" solution. ${ }^{22}$ In the following, the coefficients $b_{+}$and $b_{-}$are considered as functions of $W$ instead of $\zeta$, because, as explained in Ref. 23, the mapping between $W$ and $\zeta$ given by

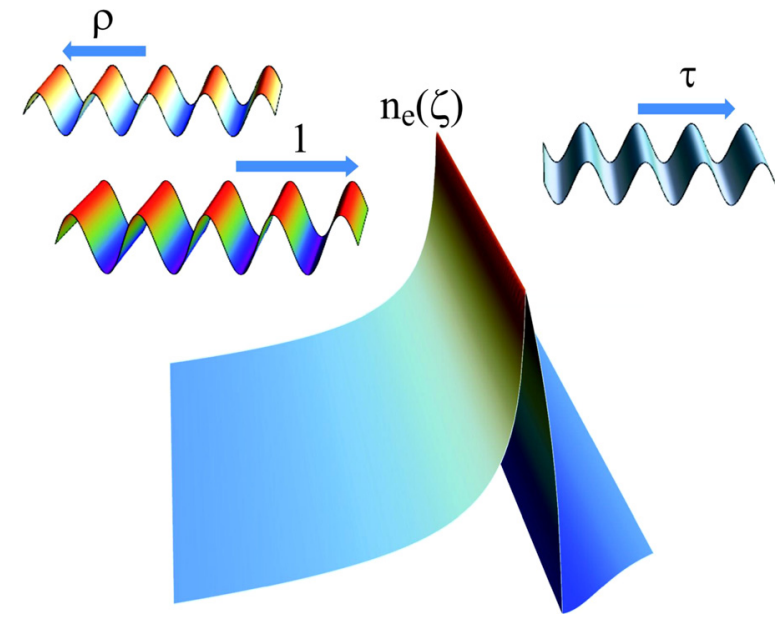

FIG. 3. Scattering geometry.

Eq. (15) is one-to-one on the real axis. Far from the breaking point, i.e., formally for $\zeta \rightarrow \pm \infty$ the function $q^{2}(\zeta) \rightarrow s^{2}$ reduces to a constant and the solutions (14) are exact, so that $b_{ \pm} \rightarrow$ const as $W \rightarrow \pm \infty$.

In other words, the boundary conditions at $\zeta \rightarrow \pm \infty$ are

$$
\begin{array}{ll}
b_{+}(-\infty)=1, & b_{-}(-\infty)=\rho, \\
b_{+}(+\infty)=\tau, & b_{-}(+\infty)=0 .
\end{array}
$$

Since in the representation (14), the single unknown function $a(\zeta)$ has been replaced by the two unknown functions $b_{ \pm}(\zeta)$, an additional condition is necessary. We shall impose the condition

$$
\frac{d a}{d \zeta}=i \sqrt{q(\zeta)}\left(b_{+} e^{i W(\zeta)}-b_{-} e^{-i W(\zeta)}\right)
$$

Differentiating Eq. (14) with respect to $\zeta$ and taking into account the constraint (17), we find

$$
\frac{d b_{+}}{d \zeta} e^{i W(\zeta)}+\frac{d b_{-}}{d \zeta} e^{-i W(\zeta)}=\frac{d \ln \sqrt{q}}{d \zeta}\left(b_{+} e^{i W(\zeta)}+b_{-} e^{-i W(\zeta)}\right)
$$

while differentiating Eq. (17) with respect to $\zeta$ and substituting $d^{2} a / d \zeta^{2}$ into Eq. (10) yields

$$
\frac{d b_{+}}{d \zeta} e^{i W(\zeta)}-\frac{d b_{-}}{d \zeta} e^{-i W(\zeta)}=\frac{d \ln \sqrt{q}}{d \zeta}\left(b_{-} e^{-i W(\zeta)}-b_{+} e^{i W(\zeta)}\right) .
$$

The system of Eqs. (18) and (19) is equivalent to Eq. (10). It can be rewritten in the form

$$
\frac{d}{d W}\left(\begin{array}{l}
b_{+} \\
b_{-}
\end{array}\right)=\left(\begin{array}{cc}
0 & S(W) e^{-2 i W} \\
S(W) e^{2 i W} & 0
\end{array}\right)\left(\begin{array}{l}
b_{+} \\
b_{-}
\end{array}\right),
$$

with

$$
S(W)=\frac{1}{2} \frac{d}{d W} \ln q(\zeta(W))
$$

Integrating both sides of Eq. (20) and using the above formulated boundary conditions for $b_{ \pm}( \pm \infty)$, we can obtain 
the reflection coefficient $\rho$ in the form of the infinite series $^{24,25}$

$$
\begin{aligned}
\rho=- & \sum_{m=0}^{\infty}(-1)^{m} \int_{-\infty}^{+\infty} d W_{0} S\left(W_{0}\right) e^{2 i W_{0}} \prod_{n=1}^{m} \int_{-\infty}^{W_{n}-1} d V_{n} S\left(V_{n}\right) e^{-2 i V_{n}} \\
& \times \int_{V_{n}}^{+\infty} d W_{n} S\left(W_{n}\right) e^{2 i W_{n}}
\end{aligned}
$$

where for $m=0$ the product is assumed to be unity.

In the case of weak reflection, $|\rho| \ll 1$, which requires $s^{2} \gg \nu(\zeta)$ in Eq. (12), the reflected wave can be found within the framework of perturbation theory. This corresponds to the known approximation in quantum mechanics considering the potential energy in the Schrödinger equation as a perturbation. ${ }^{26}$ This approximation has also been used for describing acoustic and electromagnetic wave propagation in layered media. ${ }^{27,28}$ This yields

$$
\rho=\frac{i}{2 s} \int_{-\infty}^{+\infty} \nu(\zeta) e^{-2 i s \zeta} d \zeta .
$$

\section{B. Reflection at the wave close to the wavebreaking threshold}

As an example of the calculation of the reflection coefficient, we consider a typical dependence of the electron density in the vicinity of the wavebreaking threshold ${ }^{1}$ in a cold plasma. It can be approximated by the expression

$$
n(X)=\frac{n_{0} G_{2 / 3}}{k_{p}^{2 / 3}\left(l^{2}+X^{2}\right)^{1 / 3}} .
$$

Here, $G_{2 / 3}$ is the dimensionless coefficient and the parameter $l$ shows how close the wave is to the wavebreaking limit, for which $l=0$. In the boosted frame of reference, this results in

$$
\nu(\zeta)=\frac{g_{2 / 3}}{\left(\sigma^{2}+\zeta^{2}\right)^{1 / 3}}
$$

with $\sigma=k_{p} l$ and $g_{2 / 3}=(2 / 9)^{1 / 3}\left(1+a_{m}^{2}\right)^{1 / 6} k_{p}^{4 / 3} \gamma_{p h}^{4 / 3}$ (see Eq. (44) in Ref. 1). Calculating the integral (23) for the integrand given by Eq. (25), we find

$$
\rho_{2 / 3}(s, l)=\frac{i \pi^{1 / 2} g_{2 / 3}}{s^{7 / 6} l^{5 / 6} \Gamma(1 / 3)} K_{1 / 6}(2 s l) .
$$

Here, $\Gamma(z)$ and $K_{\nu}(z)$ are the Euler gamma function and the modified Bessel function, ${ }^{29}$ respectively.
In the limit of relatively large $l$, when $s l \gg 1$, the above-barrier reflection is exponentially weak,

$$
\rho_{2 / 3}(s, l) \approx \frac{i \pi g_{2 / 3}}{s^{5 / 3} l^{2 / 3} \Gamma(1 / 3)} e^{-2 s l} .
$$

In the opposite case, when $s l \rightarrow 0$, we have a nonexponentially small reflection coefficient ${ }^{12}$

$$
\rho_{2 / 3}(s) \approx \frac{i 3^{1 / 2} \Gamma(1 / 3) g_{2 / 3}}{(2 s)^{2}} .
$$

This yields the reflection coefficient

$$
\mathrm{R}_{2 / 3}(s) \approx \frac{3 \Gamma^{2}(1 / 3) g_{2 / 3}^{2}}{(2 s)^{4}}
$$

In Fig. 4, we show the normalized amplitude of the reflected EM wave, $\rho(s)$ as a function of the normalized wavenumber $s$ calculated for different electron density $\nu(\zeta)$ profiles depending on the parameter $\sigma$ which is equal to 0.05 , $0.1,0.2,0.3,0.4,0.5$ - from the upper to bottom curves, respectively. The smaller the parameter $\sigma$ is, i.e., closeness of the wakewave to the breaking limit, the higher is the reflection. In the short wavelength limit, $s \sigma \gg 1$, the reflection is exponentially weak.

In thermal plasmas in the neighbourhood of the point where the wave breaks, $q^{2}(\zeta)$ in Eq. (12) can be written as

$$
q^{2}(\zeta)=s^{2}+g_{-1}|\zeta|
$$

i.e., the function $\nu(\zeta)$ is equal to $g_{-1}|\zeta|$. The coefficient $g_{-1}$ is equal to

$$
g_{-1}=\frac{\omega_{p e}^{2} \sqrt{n_{\mathrm{br}}}}{\Delta p_{0} c^{2} \sqrt{\gamma_{\mathrm{ph}}}} .
$$

For $q(\zeta)$ given by Eq. (30), we have

$$
W(\zeta)=\frac{2}{3 g_{-1}}\left[\left(s^{2}+g_{-1}|\zeta|\right)^{3 / 2}-s^{3}\right] \operatorname{sign}(\zeta),
$$

where $\operatorname{sign}(\zeta)=-1$ if $\zeta<0$ and $\operatorname{sign}(\zeta)=1$ for $\zeta>0$, and

$$
q(W)=\left(\frac{3 g-1}{2} W \operatorname{sign}(\zeta)+s^{3}\right)^{1 / 3}
$$

so that
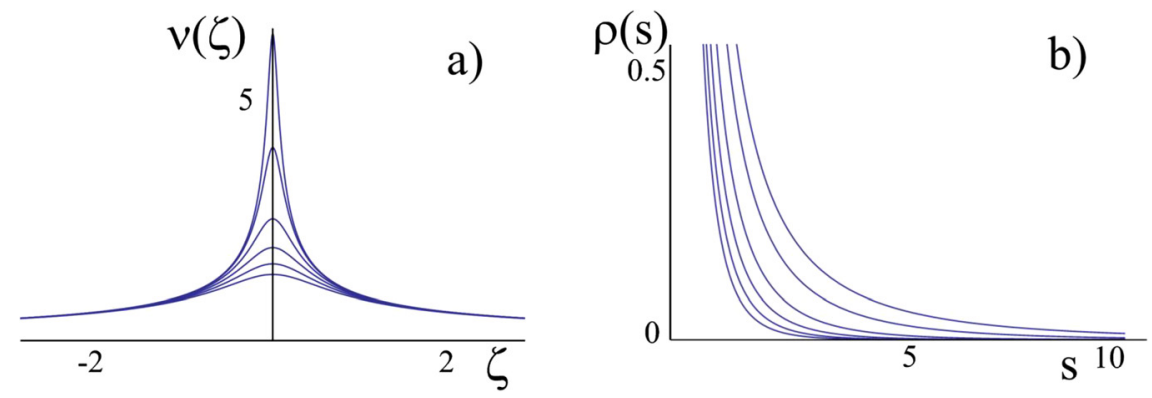

FIG. 4. (a) Normalized electron density $\nu(\zeta)=1 /$ $\left(\sigma^{2}+\zeta^{2}\right)^{1 / 3}$, (b) normalized amplitude of the reflected EM wave, $\rho(s)=i \pi^{1 / 2} s^{-7 / 6} \sigma^{5 / 6} \Gamma(1 / 3)$ $K_{1 / 6}(2 s \sigma)$, for $\sigma=0.05,0.1,0.2,0.3,0.4,0.5$ from the upper to bottom curves, respectively. 


$$
S(W)=\frac{g_{-1}}{4 q^{3}(\zeta(W))} \operatorname{sign}(\zeta) .
$$

It follows that $S(W)$ is discontinuous at $\zeta \rightarrow 0(W \rightarrow 0)$

$$
S(W=0)=\frac{g_{-1}}{4 s^{3}} \operatorname{sign}(\zeta) .
$$

This corresponds to the fact that the function $q(\zeta)$ defined by Eq. (30) has a discontinuous first derivative at $\zeta=0$. In the vicinity of the singularity point, it can be represented in the form $q(\zeta) \approx q_{0}+q_{1}|\zeta|$ with $q_{0}=s$ and $q_{1}=g_{-1} / 2 s$. Expanding $W(\zeta)$ and $S(W)$ in powers of $\zeta$ and substituting them into Eq. (22), we can find (see Eq. (27) of Ref. 23) that the first term yields the dominant contribution to the reflection coefficient, with the result

$$
\rho_{-1} \approx \frac{-i q_{1}}{s q_{0}}=\frac{-i g_{-1}}{4 s^{3}}
$$

and

$$
R_{-1}=\left|\rho_{-1}\right|^{2}=\frac{g_{-1}^{2}}{s^{6}}
$$

Applicability of the WKB theory implies that $g_{-1} \ll s^{3}$.

If we pay attention to the thermal broadening of the edges of the water-bag distribution, we shall see a smoothing of the singularity in the spatial derivative of the density. A rough estimate of the effect of this factor on the reflectivity can be made within the framework of the approximation similar to that used above for consideration of the wakewave below the breaking limit. We replace $\nu(\zeta)=g_{-1}|\zeta|$ with

$$
\nu(\zeta)=-g_{-1} \sqrt{\zeta^{2}+\sigma^{2}}
$$

where $\sigma$ is a measure of the thermal broadening of the edges of the water-bag distribution or/and its value characterizes how close the wave is to the singularity. Using expression (23), it is easy to obtain for the amplitude of the reflected wave

$$
|\rho(s)|=g_{-1} \frac{\sigma K_{1}(2 s \sigma)}{2 s^{2}} .
$$

Asymptotic expansions of the modified Bessel function $K_{1}(z)$ at $z \rightarrow 0$ and $z \rightarrow \infty$ yield the reflectivity in the long wavelength limit, $|\rho(s)| \approx g_{1} / 4 s^{3}$ for $s \sigma \ll 1$ (in accordance with Eq. (36)), and $|\rho(s)| \approx g_{1}\left(\sqrt{\pi \sigma} / 4 s^{5 / 2}\right) e^{-2 s \sigma}$ in the short wavelength limit, when $s \sigma \gg 1$.
In Fig. 5, we show the normalized amplitude of the reflected EM wave, $\rho(s)$ as a function of the normalized wavenumber $s$ calculated for different electron density $\nu(\zeta)$ profiles depending on the parameter $\sigma$ which is equal to 0.05 , $0.1,0.2,0.3,0.4,0.5$ - from the upper to bottom curves, respectively. The smaller the parameter $\sigma$ is, i.e., the smallness of the thermal broadening of the electron distribution or/and closeness of the wakewave to the breaking limit, the higher is the reflection. In the short wavelength limit, $s \sigma \gg 1$, the reflection is exponentially weak.

\section{Reflectivity in the above breaking regime}

Similarly, we can find the reflection coefficient at the electron density singularity formed in the above breaking regime discussed in Part I. $^{1}$ In this case, the electron density distribution is given by Eq. (63) of Part I. Using this relationship, we obtain

$$
\begin{aligned}
\rho_{\left(\frac{1}{2}, \frac{1}{2}\right)}= & \frac{-4 i g_{\left(\frac{1}{2}, \frac{1}{2}\right)}}{s} \int_{-\infty}^{+\infty} \exp [2 i \zeta \zeta] \\
& \times(\theta(\zeta) \sqrt{\zeta}-\theta(\zeta-\Delta \zeta) \sqrt{\zeta-\Delta \zeta}) d \zeta
\end{aligned}
$$

where

$$
g_{\left(\frac{1}{2}, \frac{1}{2}\right)}=k_{p}^{3 / 2} \gamma_{\mathrm{ph}}^{3 / 2} \frac{\sqrt{2 e E_{\max } m_{e} c}}{\Delta p_{0}}
$$

$k_{p}=c / \omega_{p e}$ and $\Delta \zeta=\Delta p_{0} / e E_{\max }$. Calculating the integral (40), we find

$$
\rho_{\left(\frac{1}{2}, \frac{1}{2}\right)}=g_{\left(\frac{1}{2}, \frac{1}{2}\right)}(1+i) \sqrt{\pi} \frac{\exp (i s \Delta \zeta) \sin (s \Delta \zeta)}{s^{5 / 2}}
$$

Consequently, we write

$$
R_{\left(\frac{1}{2}, \frac{1}{2}\right)}=\left|\rho_{\left(\frac{1}{2}, \frac{1}{2}\right)}\right|^{2}=g_{\left(\frac{1}{2}, \frac{1}{2}\right)}^{2} 2 \pi \frac{\sin ^{2}(s \Delta \zeta)}{s^{5}} .
$$

From Eqs. (37) and (43), we can see that in thermal plasmas the reflection coefficient is $s \gg 1$ times larger in the above breaking regime than for a wake wave approaching the wavebreaking threshold.

Generalizing Eqs. (63) and (65) of Part I, ${ }^{1}$ we can write the electron density dependence on the coordinate $\zeta$ in the form
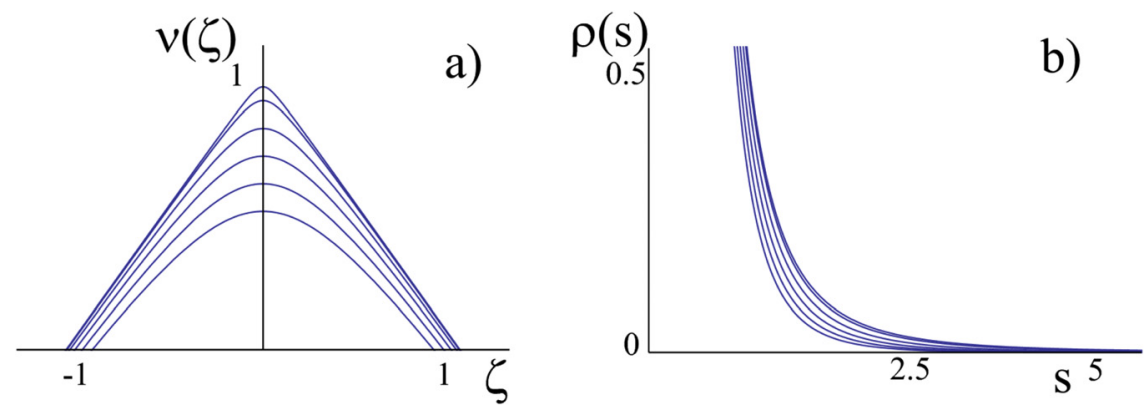

FIG. 5. (a) Normalized electron density $\nu(\zeta)=1-\left(\sigma^{2}+\zeta^{2}\right)^{1 / 2}$, (b) normalized amplitude of the reflected EM wave, $\rho(s)=\sigma K_{1}(2 s \sigma) / 2 s^{2}$, for $\sigma=0.05,0.1,0.2,0.3,0.4,0.5-$ from the upper to bottom curves, respectively. 


$$
n_{e}(\zeta) \sim \frac{2 n_{0}}{\Delta \zeta}\left[\theta\left(\zeta_{+}\right)\left(\zeta_{+}\right)^{1 / m}-\theta\left(\zeta_{-}\right)\left(\zeta_{-}\right)^{1 / m}\right]
$$

with $\zeta_{ \pm}=\zeta \pm \Delta \zeta / 2$ and $m$ an even number, and

$$
\begin{aligned}
n_{e}(\zeta) \sim & \frac{n_{0}}{\Delta \zeta}\left[\theta\left(\zeta_{+}\right)\left(\zeta_{+}\right)^{1 / m}+\theta\left(-\zeta_{-}\right)\left(-\zeta_{-}\right)^{1 / m}\right. \\
& \left.-\theta\left(\zeta_{-}\right)\left(\zeta_{-}\right)^{1 / m}-\theta\left(-\zeta_{+}\right)\left(-\zeta_{+}\right)^{1 / m}\right]
\end{aligned}
$$

for $m$ an odd number.

It is easy to show that for the reflection coefficient,

$$
\begin{aligned}
R_{\left(\frac{1}{m}, \frac{1}{m}\right)}= & \left|\rho_{\left(\frac{1}{m}, \frac{1}{m}\right)}\right|^{2} \text {, we have } \\
& R_{\left(\frac{1}{m}, \frac{1}{m}\right)}=g_{\left(\frac{1}{m}, \frac{1}{m}\right)}^{2} 2^{8}\left[\frac{\Gamma(1+1 / m)}{(2 s)^{1+1 / m}} \frac{\sin (s \Delta \zeta)}{s \Delta \zeta}\right]^{2},
\end{aligned}
$$

if $m$ is even, and

$$
R_{\left(\frac{1}{m}, \frac{1}{m}\right)}=g_{\left(\frac{1}{m}, \frac{1}{m}\right)}^{2} 2^{7}\left(1+\cos \frac{\pi}{m}\right)\left[\frac{\Gamma(1+1 / m)}{(2 s)^{1+1 / m}} \frac{\sin (s \Delta \zeta)}{s \Delta \zeta}\right]^{2},
$$

if $m$ is odd.

Comparing Eqs. (43), (46), and (47) for the reflection coefficient with the corresponding coefficients obtained in Ref. 12 , we find that the effects of a finite temperature enter Eqs. (43), (46), and (47) as a form-factor $[\sin (s \Delta \zeta) / s \Delta \zeta]^{2}$. In the limit $\Delta p_{0} \rightarrow 0$, this form factor tends to unity.

Since the frequency, $\omega_{r}$, and the number of reflected photons, $N_{r}$, are related to that incident on the relativistic mirror, $\omega_{0}$ and $N_{s}$, as $\omega_{r}=\omega_{0}\left(1+\beta_{\mathrm{ph}}\right) /\left(1-\beta_{\mathrm{ph}}\right) \approx \omega_{0} 4 \gamma_{\mathrm{ph}}^{2}$ and $N_{r}=R N_{s}$, the energy of the reflected photon beam is given by $\mathcal{E}_{r} \approx \mathcal{E}_{s} 4 \gamma_{\mathrm{ph}}^{2} R$, where $\mathcal{E}_{s}$ is the energy of the laser pulse incident on the mirror. Comparing $\mathcal{E}_{r}$ with the energy of the electrons in the first period of the wake wave (e.g., see Ref. 30), $\mathcal{E}_{e} \approx \mathcal{E}_{\text {las }, \mathrm{d}}\left(\omega_{\text {pe }} / \omega_{0}\right)^{2}$, where $\mathcal{E}_{\text {las }, \mathrm{d}}$ is the laser driver energy, we find that the photon back reaction (the ponderomotive pressure) on the wake wave can be neglected provided $\mathcal{E}_{s} \ll \mathcal{E}_{\text {las }, \mathrm{d}} / 4 \gamma_{\mathrm{ph}}^{4} R$. As a typical reflection coefficient value, we can take $R \approx 0.1 / \gamma_{\text {ph }}^{4}$ (see Refs. 9 and 12) and obtain the condition of relative weakness of the incident laser pulse $\mathcal{E}_{s} \leq \mathcal{E}_{\text {las }, \mathrm{d}}$. As we see owing to the weakness of the photon-wakewave interaction, the incident pulse energy can be of the order of that in the driver laser pulse.

The wake wave can be typically comprised of a sequence of spikes with some spatial period as discussed in Part-I. ${ }^{1}$ The above-barrier reflectivity from such the structures has been analyzed in Refs. 12 and 31 and demonstrated with computer simulations in Refs. 32 and 33. The counterpropagating pulse experiences multiple reflections giving rise to a train of ultrashort pulses. While the number of photons reflected at each layer, $N_{r}=R N_{s}$, where $N_{s}$ is the number of photons in the counterpropagating pulse, is small, the multiple spikes can reflect almost all incoming photons, $N_{r} \rightarrow N_{s}$, when the number of spikes, $N_{w}$, is large enough, $N_{w} \gg 1 / R$. The total reflected energy tends to $\mathcal{E}_{r}=4 \gamma^{2} \mathcal{E}_{s}$ in this limit. Here, the interaction of the outgoing wave with the density spikes is neglected, because the reflecting structure moves with relativistic velocity. During the first reflection, a portion of the (counter-propagating) source pulse is shortened by a factor $\approx 4 \gamma_{\mathrm{ph}}^{2}$, e.g., under the conditions of the experiments ${ }^{8-10}$ by a factor about 100, and typically the reflected pulse becomes much shorter than the wake wave wavelength, which is equal to the distance between subsequent peaks. The time for the outgoing wave to reach the next peak is of the order of $\omega_{\mathrm{pe}}^{-1} /\left(1-\beta_{\mathrm{ph}}\right) \approx 2 \omega_{\mathrm{pe}}^{-1} \gamma_{\mathrm{ph}}^{2}$, which is of the order of the driver laser pulse energy depletion time. Due to this, the time required for reflections of the outgoing wave from the density spikes is well above the laser pulse energy depletion time.

\section{CONCLUSION}

In the first Part of our paper, ${ }^{1}$ we found the structure of the typical singularities that appear in the electron density during the wave breaking in a thermal plasma. The singularity in the electron density, moving along with the wake wave excited by a high intensity ultra-short pulse laser, can act as a flying relativistic mirror for counterpropagating electromagnetic radiation, leading to coherent reflection accompanied by the upshift of the radiation frequency. This process implies finite (not exponentially small) reflectivity at the electron density singularities. This is provided by the structure of the singularity formed in a relativistically large amplitude plasma wave close to the wavebreaking limit that leads to a refraction coefficient with discontinuous spatial derivatives. We found the reflection coefficients of an electromagnetic wave at the singularities of the electron density in the most typical regimes of strongly nonlinear wave breaking in thermal plasmas. The efficiency of the photon reflection can be substantially increased using the above breaking limit regimes which lead to the formation of highorder singularities.

\section{ACKNOWLEDGMENTS}

We thank M. Lobet for discussions. We acknowledge the support from the MEXT of Japan, Grant-in-Aid for Scientific Research, 23740413, and Grant-in-Aid for Young Scientists 21740302 from MEXT. We appreciate support from the NSF under Grant No. PHY-0935197 and the Office of Science of the US DOE under Contract No. DE-AC02$05 \mathrm{CH} 11231$.

${ }^{1}$ S. V. Bulanov, T. Zh. Esirkepov, M. Kando, J. K. Koga, A. S. Pirozhkov, T. Nakamura, S. S. Bulanov, C. B. Schroeder, E. Esarey, F. Califano, and F. Pegoraro, Phys. Plasmas 19, 113102 (2012).

${ }^{2}$ R. C. Davidson, Methods in Nonlinear Plasma Theory (Academic, New York, 1972).

${ }^{3}$ G. G. Stokes, Trans. Cambridge Philos. Soc. 8, 441 (1847); G. G. Stokes, Mathematical and Physical Papers (Cambridge, 1880), Vol. I, pp. 197219; J. Wilkening, Phys. Rev. Lett. 107, 184501 (2011).

${ }^{4}$ G. B. Whitham, Linear and Nonlinear Waves (Wiley-Interscience, New York, 1974).

${ }^{5}$ R. Camassa and D. D. Holm, Phys. Rev. Lett. 71, 1661 (1993); A. Degasperis and M. Procesi, in Symmetry and Perturbation Theory //, edited by A. Degasperis and G. Gaeta (World Scientific, River Edge, NJ, 1999), pp. 23-37. 
${ }^{6}$ S. V. Bulanov, I. N. Inovenkov, V. I. Kirsanov, N. M. Naumova, and A. S. Sakharov, Sov. Phys. Lebedev. Inst. Rep. 6, 9 (1991) [Kratk. Soobshch. Fiz. 6, 9 (1991)]; S. V. Bulanov, F. Califano, G. I. Dudnikova, T. Zh. Esirkepov, I. N. Inovenkov, F. F. Kamenets, T. V. Liseikina, M. Lontano, K. Mima, N. M. Naumova, K. Nishihara, F. Pegoraro, H. Ruhl, A. S. Sakharov, Y. Sentoku, V. A. Vshivkov, and V. V. Zhakhovskii, Reviews of Plasma Physics, edited by V. D. Shafranov (Kluwer Academic, New York, 2001), Vol. 22, p. 227.

${ }^{7}$ S. V. Bulanov, T. Zh. Esirkepov, and T. Tajima, Phys. Rev. Lett. 91, 085001 (2003).

${ }^{8}$ M. Kando, Y. Fukuda, A. S. Pirozhkov, J. Ma, I. Daito, L.-M. Chen, T. Zh. Esirkepov, K. Ogura, T. Homma, Y. Hayashi, H. Kotaki, A. Sagisaka, M. Mori, J. K. Koga, H. Daido, S. V. Bulanov, T. Kimura, Y. Kato, and T. Tajima, Phys. Rev. Lett. 99, 135001 (2007).

${ }^{9}$ A. S. Pirozhkov, J. Ma, M. Kando, T. Zh. Esirkepov, Y. Fukuda, L.-M. Chen, I. Daito, K. Ogura, T. Homma, Y. Hayashi, H. Kotaki, A. Sagisaka, M. Mori, J. K. Koga, T. Kawachi, H. Daido, S. V. Bulanov, T. Kimura, Y. Kato, and T. Tajima, Phys. Plasmas 14, 123106 (2007).

${ }^{10}$ M. Kando, A. S. Pirozhkov, K. Kawase, T. Zh. Esirkepov, Y. Fukuda, H. Kiriyama, H. Okada, I. Daito, T. Kameshima, Y. Hayashi, H. Kotaki, M. Mori, J. K. Koga, H. Daido, A. Ya. Faenov, T. Pikuz, J. Ma, L.-M. Chen, E. N. Ragozin, T. Kawachi, Y. Kato, T. Tajima, and S. V. Bulanov, Phys. Rev. Lett. 103, 235003 (2009).

${ }^{11}$ S. S. Bulanov, T. Zh. Esirkepov, F. F. Kamenets, and F. Pegoraro, Phys. Rev. E 73, 036408 (2006); S. S. Bulanov, A. Maximchuk, C. B. Schroeder, A. G. Zhidkov, E. Esarey, and W. P. Leemans, Phys. Plasmas 19, 020702 (2012).

${ }^{12}$ A. V. Panchenko, T. Zh. Esirkepov, A. S. Pirozhkov, M. Kando, F. F. Kamenets, and S. V. Bulanov, Phys. Rev. E 78, 056402 (2008).

${ }^{13}$ S. C. Wilks, J. M. Dawson, W. B. Mori, T. Katsouleas, and M. E. Jones, Phys. Rev. Lett. 62, 2600 (1989).

${ }^{14}$ J. T. Mendonca, Photon Acceleration in Plasmas (IOP, Bristol, 2001).

${ }^{15}$ V. P. Silin, Sov. Phys. JETP 11, 1136 (1960); B. Kurşunoğlu, Nucl. Fusion 1, 213 (1961); A. B. Mikhajlovskii, Plasma Phys. 22, 133 (1980); D. B. Melrose, Aust. J. Phys. 35, 41 (1982); J. Bergman and B. Eliasson, Phys. Plasmas 8, 1482 (2001)

${ }^{16}$ V. A. Mironov, A. M. Sergeev, E. V. Vanin, and G. Brodin, Phys. Rev. A 42, 4862 (1990); S. V. Bulanov and A. S. Sakharov, JETP Lett. 54, 203 (1991); V. A. Mironov, A. M. Sergeev, E. V. Vanin, G. Brodin, and J. Lundberg, Phys. Rev. A 46, R6178 (1992); J. T. Mendonca and L. O. Silva, Phys. Rev. E 49, 3520 (1994); R. Bingham, J. T. Mendonca, and J. M. Dawson, Phys. Rev. Lett. 78, 247 (1997); L. O. Silva and J. T. Mendonca, Phys. Rev. E 57, 3423 (1998); A. A. Solodov, P. Mora, and P. Chessa, Phys. Plasmas 6, 503 (1999); A. Spitkovsky and P. Chen, Phys. Lett. A 296, 125 (2002); G. Raj, M. R. Islam, B. Ersfeld, and D. A. Jaroszynski, Phys. Plasmas 17, 073102 (2010).

${ }^{17}$ C. W. Siders, S. P. LeBlanc, D. Fisher, T. Tajima, M. C. Downer, A. Babine, A. Stepanov, and A. Sergeev, Phys. Rev. Lett. 76, 3570 (1996); J.
M. Dias, C. Stenz, N. Lopes, X. Badiche, F. Blasco, A. Dos Santos, L. O. Silva, A. Mysyrowicz, A. Antonetti, and J. T. Mendonca, Phys. Rev. Lett. 78, 4773 (1997); C. D. Murphy, R. Trines, J. Vieira, A. J. W. Reitsma, R. Bingham, J. L. Collier, E. J. Divall, P. S. Foster, C. J. Hooker, A. J. Langley, P. A. Norreys, R. A. Fonseca, F. Fiuza, L. O. Silva, J. T. Mendonca, W. B. Mori, J. G. Gallacher, R. Viskup, D. A. Jaroszynski, S. P. D. Mangles, A. G. R. Thomas, K. Krushelnick, and Z. Najmudin, Phys. Plasmas 13, 033108 (2006); R. M. G. M. Trines, C. D. Murphy, K. L. Lancaster, O. Chekhlov, P. A. Norreys, R. Bingham, J. T. Mendonca, L. O. Silva, S. P. D. Mangles, C. Kamperidis, A. Thomas, K. Krushelnick, and Z. Najmudin, Plasma Phys. Controlled Fusion 51, 024008 (2009).

${ }^{18}$ T. Esirkepov, S. V. Bulanov, M. Yamagiwa, and T. Tajima, Phys. Rev. Lett. 96, 014803 (2006).

${ }^{19}$ A. Einstein, Ann. Phys. (Leipzig) 17, 891 (1905).

${ }^{20}$ V. L. Pokrovskii, S. K. Savinykh, and F. R. Ulinich, Sov. Phys. JETP 34, 879 (1958); V. L. Pokrovskii and I. M. Khalatnikov, Sov. Phys. JETP 13, 1207 (1961).

${ }^{21}$ J. T. Mendonca and K. Hizanidis, Plasma Phys. Controlled Fusion 54, 035006 (2012).

${ }^{22}$ N. Fröman and P. O. Fröman, JWKB Approximation: Contributions to the Theory (North-Holland P.C., Amsterdam, 1965).

${ }^{23}$ M. V. Berry, J. Phys. A 15, 3693 (1982).

${ }^{24}$ M. V. Berry and K. E. Mount, Rep. Prog. Phys. 35, 315 (1972).

${ }^{25}$ H. Bremmer, Commun. Pure Appl. Math. 4, 105 (1951); R. Landauer, Phys. Rev. 82, 80 (1951).

${ }^{26}$ L. D. Landau and E. M. Lifshitz, Quantum Mechanics Non-Relativistic Theory (Butterworth and Heinemann, Oxford, 1997).

${ }^{27}$ L. M. Brekhovskikh, Waves in Layered Media (Academic, New York, 1980).

${ }^{28}$ V. L. Ginzburg, The Propagation of Electromagnetic Waves in Plasmas (Pergamon, Oxford, 1970).

${ }^{29}$ M. Abramowitz and I. A. Stegun, Handbook of Mathematical Functions with Formulas, Graphs, and Mathematical Tables (Dover, New York, 1964).

${ }^{30}$ E. Esarey, C. B. Schroeder, and W. P. Leemans, Rev. Mod. Phys. 81, 1229 (2009).

${ }^{31}$ S. V. Bulanov, L.-M. Chen, H. Daido, I. Daito, T. Zh. Esirkepov, Y. Fukuda, Y. Hayashi, T. Homma, M. Kando, T. Kimura, J. K. Koga, H. Kotaki, J. Ma, M. Mori, K. Ogura, A. S. Pirozhkov, A. Sagisaka, and T. Tajima, in Proceedings of the International Workshop on Quark Nuclear Physics, edited by J. K. Ahn, M. Fujiwara, T. Hayakawa, A. Hosaka, T. Hotta, H. C. Kim, T. Myo, K. Sumiyoshi, and S.-L. Zhu (Pusan National Univesity Press, PUSAN, 2006), pp.179-194.

${ }^{32}$ S. S. Bulanov, A. Maksimchuk, K. Krushelnick, K. I. Popov, V. Y. Bychenkov, and W. Rozmus, Phys. Lett. A 374, 476 (2010).

${ }^{33}$ M. Lobet, M. Kando, J. K. Koga, T. Zh. Esirkepov, T. Nakamura, A. S. Pirozhkov, and S. V. Bulanov, e-print arXiv:1209.3912. 